\title{
Prostate Osteoblast-Like Cells: A Reliable Prognostic Marker of Bone Metastasis in Prostate Cancer Patients
}

\author{
Manuel Scimeca $\mathbb{D}^{1,2}$ Nicoletta Urbano, ${ }^{3}$ Bonfiglio Rita ${ }^{1},{ }^{4}$ Sarah Natalia Mapelli, ${ }^{5}$ \\ Carlo Vittorio Catapano, ${ }^{5}$ Giuseppina Maria Carbone, ${ }^{5}$ Sara Ciuffa, ${ }^{4}$ Mario Tavolozza, ${ }^{3}$ \\ Orazio Schillaci, ${ }^{1,6}$ Alessandro Mauriello (i), ${ }^{4}$ and Elena Bonanno (iD) \\ ${ }^{1}$ Department of Biomedicine and Prevention, University of Rome "Tor Vergata", Via Montpellier 1, Rome 00133, Italy \\ ${ }^{2}$ University San Raffaele, Via di Val Cannuta 247, 00166 Rome, Italy \\ ${ }^{3}$ Nuclear Medicine, Policlinico "Tor Vergata”, Rome, Italy \\ ${ }^{4}$ Department of Experimental Medicine and Surgery, University "Tor Vergata", Via Montpellier 1, Rome 00133, Italy \\ ${ }^{5}$ Università della Svizzera Italiana (USI), Institute of Oncology Research (IOR), Via Vela 6, Bellinzona, Switzerland \\ ${ }^{6}$ IRCCS Neuromed, Pozzilli, IS, Italy \\ ${ }^{7}$ IRCCS Neuromed Lab. "Diagnostica Medica" and "Villa dei Platani", Avellino, Italy
}

Correspondence should be addressed to Elena Bonanno; elena.bonanno@uniroma2.it

Received 10 July 2018; Accepted 20 November 2018; Published 9 December 2018

Academic Editor: Ralf Schirrmacher

Copyright (c) 2018 Manuel Scimeca et al. This is an open access article distributed under the Creative Commons Attribution License, which permits unrestricted use, distribution, and reproduction in any medium, provided the original work is properly cited.

\begin{abstract}
The main aim of this study was to investigate the putative association among the presence of prostate cancer cells, defined as prostate osteoblast-like cells (POLCs), and showing the expression of typical morphological and molecular characteristics of osteoblasts, the development of bone metastasis within 5 years of diagnosis, and the uptake of $18 \mathrm{~F}$-choline evaluated by PET/CT analysis. To this end, prostate biopsies $(n=110)$ were collected comprising 44 benign lesions and 66 malignant lesions. Malignant lesions were further subdivided into two groups: biopsies from patients that had clinical evidence of bone metastasis $(\mathrm{BM}+, n=23)$ and biopsies from patients that did not have clinical evidence of bone metastasis within 5 years $(\mathrm{BM}-, n=43)$. Paraffin serial sections were obtained from each specimen to perform histological classifications and immunohistochemical (IHC) analysis. Small fragments of tissue were used to perform ultrastructural and microanalytical investigations. IHC demonstrated the expression of markers of epithelial-to-mesenchymal transition (VIM), bone mineralization, and osteoblastic differentiation (BMP-2, PTX-3, RUNX2, RANKL, and VDR) in prostate lesions characterized by the presence of calcium-phosphate microcalcifications and high metastatic potential. Ultrastructural studies revealed the presence of prostate cancer cells with osteoblast phenotype close to microcalcifications. Noteworthy, PET/CT analysis showed higher uptake of $18 \mathrm{~F}$-choline in BM+ lesions with high positivity ( $\geq 300 / 500$ cells) for RUNX2 and/or RANKL immunostaining. Although these data require further investigations about the molecular mechanisms of POLCs generation and role in bone metastasis, our study can open new and interesting prospective in the management of prostate cancer patients. The presence of POLCs along with prostate microcalcifications may become negative prognostic markers of the occurrence of bone metastases.
\end{abstract}

\section{Introduction}

Metastasis to bone is a common feature in advanced prostate cancer ( $\mathrm{PCa}$ ) patients. $\mathrm{PCa}$ is one of the most frequent cancer in men and represents a great public health problem, with a total of 265,000 new diagnosis every year in both Europe and United States of America [1]. Frequently, prostate cancer patients show bone osteoblastic metastatic lesions at diagnosis $[1,2]$. The evidence that prostate cancer cells in patients enter the circulation in large numbers but still preferentially colonise to the bone has a number of implications. Prostate cancer cells have the ability to adhere at the main proteins of the extracellular matrix or at the bone marrow [2]. Also, the colonisation bone by prostate cancer 
cells suggests that metastatic cells have morphological and/ or molecular characteristics that make them capable to survive in the bone [2-4].

The type of bone metastases formed in prostate cancer is a reflection of the local interaction between tumour cells and the bone remodeling system-a complex mechanism which remains to be fully characterized. Bone metastases in prostate cancer are most often osteoblastic (involving the deposition of newly formed bone), but can also be osteolytic (characterized by destruction of normal bone) or mixed. The development of either osteolytic or osteoblastic lesions results from functional interactions between tumour cells and osteoclasts or osteoblasts, respectively [5]. However, the mechanisms responsible for the formation of prostate cancer metastasis to bone are complex and certainly involve both osteoclasts and osteoblasts activity [6]. In this context, the binary classification between osteoblastic and osteolytic lesions represents two extremes of a continuum which involves dysregulation of the normal bone remodeling process and which is yet to be fully understood. A detailed characterization of the osteoblastic-osteolytic spectrum and of premetastatic tumour cells could therefore pave the way for both the identification of early markers for bone metastasis and of novel drug targets to improve quality of life of patients with advanced prostate cancer.

As concerns the origin of metastatic cells, different hypotheses have been formulated. For a long time, the main theories of the formation of bone metastases contemplated the occurrence of specific genetics change in primary cancer cells that thus acquired the ability to spread to and thrive in distant organs $[7,8]$. In this context, the epithelial-tomesenchymal transition (EMT) could represent the key biological process adopted by epithelial cancer cells to promote tissue dissemination [9]. On note, in our recent study, we demonstrated a putative association between the occurrence of EMT and the development of breast cancer cells showing an osteoblast-like cells phenotype in lesions with microcalcifications [10, 11]. In addition, we observed that the presence of breast osteoblast-like cells (BOLCs) in breast infiltrating cancer was associated with the formation of bone metastatic lesions within 5 years from diagnosis $[12,13]$.

The main aim of this study was to investigate the putative association among the presence of prostate cancer cells, defined as prostate osteoblast-like cells (POLCs), and showing the expression of typical morphological and molecular characteristics of osteoblasts, the development of bone metastasis within 5years of diagnosis, and the uptake of ${ }^{18} \mathrm{~F}$-choline evaluated by PET/CT analysis.

\section{Materials and Methods}

2.1. Collection of Prostate Samples. In this study, we enrolled 110 patients undergoing prostate biopsies. From this selection, we collected prostate biopsies from each patient and, when available, data of PET/CT analysis. The study was approved by Institutional Ethical Committee of the "Policlinico Tor Vergata." Experimental procedures here reported were performed in agreement with the The Code of Ethics of the World Medical Association (Declaration of Helsinki).
All patients have signed the informed consent prior to surgical procedures. From each sample, paraffin serial sections were used for both histological and immunohistochemical investigation. Also, $1 \mathrm{~mm}^{3}$ of tissue were studied by transmission electron microscopy and microanalytical analysis. Exclusion criteria were history of previously or concomitant other neoplastic diseases, autoimmune diseases, viral chronic infections (HBV, HCV, and HIV), and any antitumoral treatment received before biopsy.

2.2. Histology. Fixation and haematoxylin and eosin staining were performed as previously described [14].

2.3. Immunohistochemistry. To study the immunophenotypical profile of prostate metastatic cells, we performed immunohistochemical reactions to investigate the expression of the following biomarkers: vimentin (EMT), BMP-2, PTX-3, RUNX2, RANKL, and VDR (mineralization process). For antigen retrieval, $3 \mu \mathrm{m}$ thick paraffin sections were treated with citrate $\mathrm{pH} 6.0$ or EDTA citrate $\mathrm{pH} 7.8$ buffers $\left(95^{\circ} \mathrm{C}\right.$ for $\left.30 \mathrm{~min}\right)$. Then, primary antibodies listed in Table 1 were incubated for 1 hour at room temperature. HRP-DAB Detection Kit (UCS Diagnostic, Rome, Italy) was used to reveal the reaction of primary antibodies with their specific target. Immunohistochemical signal was assessed independently by two investigators by counting the number of positive cancer cells (out of a total of 500 in randomly selected regions).

2.4. Transmission Electron Microscopy (TEM) and Energy Dispersive X-Ray (EDX) Microanalysis. Small fragment of prostate tissue $\left(1 \mathrm{~mm}^{3}\right)$ was fixed in $4 \%$ paraformaldehyde and postfixed in $2 \%$ osmium tetroxide. Then, the sample was dehydrated in alcohol and infiltrated with propylene oxide before being embedded in Epon (Agar Scientific, Stansted CM24 8GF, Essex, United Kingdom) [15]. Eightymicrometer ultrathin sections were cut by ultramicrotome and mounted on copper grids. All samples were examined with a transmission electron microscope (Model JEM-1400, JEOL) [16-18].

For EDX microanalysis, $80 \mu \mathrm{m}$ ultrathin sections were mounted on copper grids. Hydroxyapatite crystals were identified by EDX detector (Thermo Scientific, Waltham, MA, USA) at an acceleration voltage of $75 \mathrm{KeV}$ and magnification of 12.000 [16-18]".

2.5. 18F-Choline PET/CT Analysis. Among patients enrolled in the study, 11 were subjected to ${ }^{18} \mathrm{~F}$-methylcholine $\left({ }^{18} \mathrm{~F}\right.$ choline) PET/CT analysis. Results of $18 \mathrm{~F}$-choline PET/CT were collected to verify a possible correlation between ${ }^{18} \mathrm{~F}$ choline uptake in prostate tumours and the presence of POLCs. ${ }^{18} \mathrm{~F}$-choline PET/CT analysis was performed as previously described $[19,20]$. From each patient, standardized uptake value (SUV) max and SUV average were recorded. 
TABLE 1: List of primary antibodies.

\begin{tabular}{lccc}
\hline Antibody & Characteristics & Dilution & Retrieval \\
\hline Antivimentin & Mouse monoclonal clone V9; Ventana, Tucson, AZ, USA & Prediluted & EDTA citrate pH 7.8 \\
Anti-BMP-2 & Rabbit monoclonal clone N/A; Novus Biologicals, Littleton, CO, USA & $1: 250$ & Citrate pH 6.0 \\
Anti-PTX-3 & Rat monoclonal clone MNB1; AbCam, Cambridge, UK & $1: 100$ & Citrate pH 6.0 \\
Anti-RUNX2 & Mouse monoclonal clone EPR14334; AbCam, Cambridge, UK & $1: 100$ & Citrate pH 6.0 \\
Anti-RANKL & Rabbit monoclonal clone 12A668; AbCam, Cambridge, UK & $1: 100$ & EDTA citrate pH 7.8 \\
Anti-VDR & Rabbit polyclonal clone NBP1-19478; Novus Biologicals, Littleton, CO, USA & $1: 100$ & Citrate pH 6.0 \\
\hline
\end{tabular}

2.6. Retrieval and Analysis of Gene Expression Datasets. Gene expression data from two studies in prostate cancer patients $[21,22]$ were retrieved from the cBioPortal platform. Expression of the selected genes was compared between primary tumours and metastatic CRPC and, for the second dataset, among primary and different metastatic sites. Heatmaps show the results of unsupervised hierarchical clustering based on the gene set expression.

2.7. Statistical Analysis. We performed groupwise comparisons of the expression of analysed biomarkers through nonparametric Kruskal-Wallis test (KW) $(p<0.05)$. Post hoc testing was performed by the Mann-Whitney test [12].

\section{Results}

3.1. Histological Classification. Prostate biopsies were classified in 44 benign lesions (BL) and 66 malignant lesions according to EAU-ESTRO-SIOG Guidelines 2017 [23]. We subdivided the malignant lesions in those $(\mathrm{BM}+, n=23)$ taken from patients with clinical evidence of bone metastasis and those (BM-, $n=43)$ from patients without clinical evidence of bone metastasis after 5 years from diagnosis. From radiological point of view, all metastatic sites showed typical characteristics of osteoblastic lesions. Calcifications were present in 38 out of 110 prostate biopsies. In particular, we observed psammoma bodies in $32 \%$ of $\mathrm{BL}, 87 \%$ of $\mathrm{BM}+$, and $79 \%$ of $\mathrm{BM}-$. Patient baseline characteristics are reported in Table 2.

3.2. EMT Characterization. Immunohistochemical analysis of vimentin expression was performed in order to evaluate the number of prostate cells that acquire mesenchymal phenotype (Figure 1(a)-1(c)). As shown in a recent study [24], significant group effect was detected in the rate of vimentin-positive prostate cells $(p=0.0025)$, and post hoc testing showed a significantly higher rate of vimentinpositive prostate cells in $\mathrm{BM}+(274.4 \pm 30.76)$ compared to both BL $(90.79 \pm 14.82)$ and $\mathrm{BM}-(198.8 \pm 22.8)$ (BL vs $\mathrm{BM}-p=0.0298 ; \mathrm{BL}$ vs $\mathrm{BM}+p<0.0001 ; \mathrm{BM}-$ vs $\mathrm{BM}+$ $p=0.047)$.

3.3. Expression of Bone Markers in Prostate Tissues. Our results showed a significant group effect on BMP-2 expression $(p=0.0494)$, and post hoc testing showed increased BMP-2 expression in BM- $(349.8 \pm 13.13)$ compared to $\mathrm{BL}(\mathrm{BL} 209.2 \pm 39.22) \quad(\mathrm{BL}$ vs $\mathrm{BM}-p=0.0083)$ (Figures 1(d)-1(f)). No other significant differences were
TABLE 2: Baseline characteristics of patients.

\begin{tabular}{lccccccc}
\hline & $\mathrm{n}$ & $\begin{array}{c}\text { Age } \\
\leq 55\end{array}$ & $\begin{array}{c}\text { Age } \\
\geq 55\end{array}$ & $\begin{array}{c}\text { Gleason } \\
\leq 6\end{array}$ & $\begin{array}{c}\text { Gleason } \\
7\end{array}$ & $\begin{array}{c}\text { Gleason } \\
\geq 8\end{array}$ & $\begin{array}{c}\text { PSA } \\
\left(\mathrm{ng} \mathrm{ml}^{-1}\right)\end{array}$ \\
\hline $\mathrm{BL}$ & 44 & 20 & 24 & $/$ & $/$ & $/$ & $\begin{array}{c}/ \\
\mathrm{BM}+\end{array}$ \\
$\mathrm{BM}-$ & 5 & 18 & 4 & 4 & 15 & $\begin{array}{c}122.11 \pm \\
1348.02\end{array}$ \\
$\mathrm{BM}$ & 13 & 30 & 13 & 6 & 25 & $\begin{array}{c}1001.09 \pm \\
147938\end{array}$ \\
\hline
\end{tabular}

found $(\mathrm{BM}+335.1 \pm 22.05)$. Our results show that prostate cells, especially cancer cells, express PTX-3, an innateimmune protein. We observed a significant group effect on PTX-3 ( $p=0.0076)$. Post hoc testing showed very strong expression of PTX-3 in the cytoplasm of BM+ (321.77 \pm 21.10) compared to both $\mathrm{BL}(154.1 \pm 23.16)$ and to BM$(205.8 \pm 28.51)(\mathrm{BL}$ vs $\mathrm{BM}-p=0.2205$; $\mathrm{BL}$ vs $\mathrm{BM}+$ $p<0.0001 ; \mathrm{BM}-$ vs $\mathrm{BM}+p=0.0158$ ) (Figures $1(\mathrm{~g})-1(\mathrm{i})$ ).

3.4. Immunophenotypic Characterization of POLCs. A significant group effect was observed on the number of RUNX2-positive cancer cells $(p=0.0187)$, after which post hoc testing showed significantly in BM- $(375.4 \pm 20.97)$ respect to $\mathrm{BL}(273.3 \pm 27.14)(p=0.0006)$. No other significant differences were found $(\mathrm{BM}+338.2 \pm 31.84)$ (Figures 2(a)-2(c)). RANKL exhibited a significant group effect $(p<0.0001)$, and in post hoc testing, its expression was significantly higher in $\mathrm{BM}+(386.7 \pm 32.26)$ with respect to both BL $(169.6 \pm 25.74)$ and BM- $(278.3 \pm 15.24)(\mathrm{BM}+\mathrm{vs}$ $\mathrm{BL} p=0.0011 ; \mathrm{BM}+\mathrm{vs} \mathrm{BM}-p=0.0014$ ) (Figures 2(d)2(f)). Also, significant differences were observed by comparing $\mathrm{BL}$ and $\mathrm{BM}-$ groups $(p<0.0001)$. VDR exhibited a similar group effect $(p<0.0001)$ (Figure $2(\mathrm{~g})-2(\mathrm{i}))$. In addition, we detected significantly higher VDR expression when comparing $\mathrm{BM}+(357 \pm 25.59)$ to both $\mathrm{BL}(3.58 \pm 2.03)$ and $\mathrm{BM}-(229.5 \pm 15.55)$ (BL vs BM- $p<0.0001 ; \mathrm{BL}$ vs $\mathrm{BM}+$ $p<0.0001 ; \mathrm{BM}-\mathrm{vs} \mathrm{BM}+p=0.0002$ ) (Figure 2(g)). In particular, the signal in $\mathrm{BM}+$ appeared very intense both in nucleus and in cytoplasm (Figure 2(h)), while it was less intense and mainly nuclear in BM- (Figure 2(i)).

3.5. Expression of POLC Biomarkers in Gene Expression Datasets. We examined expression of the EMT and bone markers studied by IHC in public datasets comprising gene expression profiling data from patients with primary tumours or metastatic castration-resistant prostate cancer (CRPC). Individual gene comparisons did not show a univocal behaviour (Figure 3(a)). Only VDR expression was 


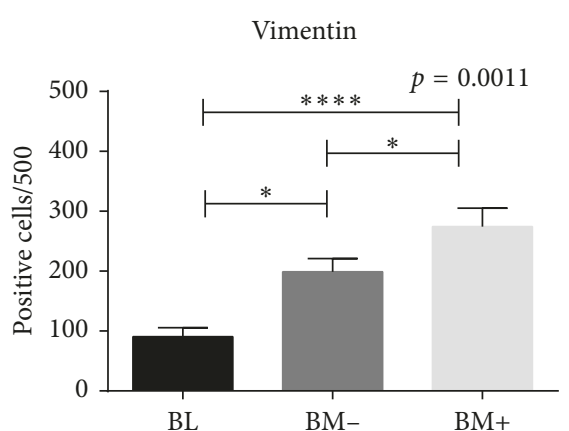

(a)

BMP-2

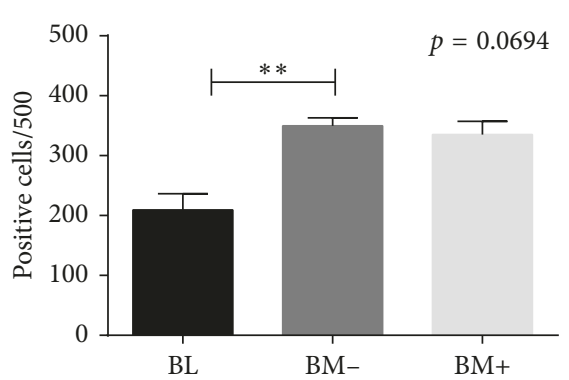

(d)

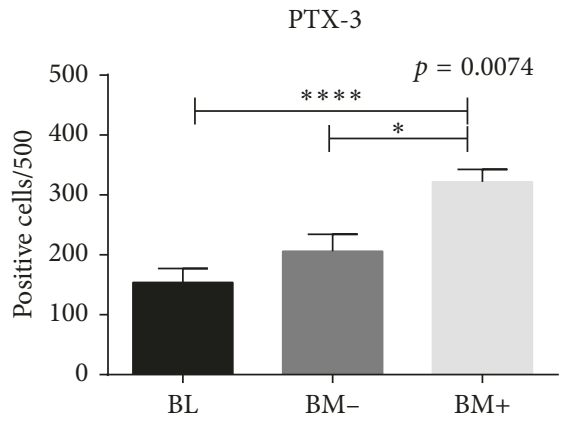

(g)

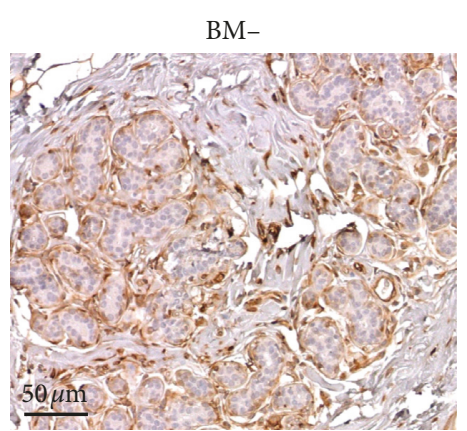

(b)

BM-

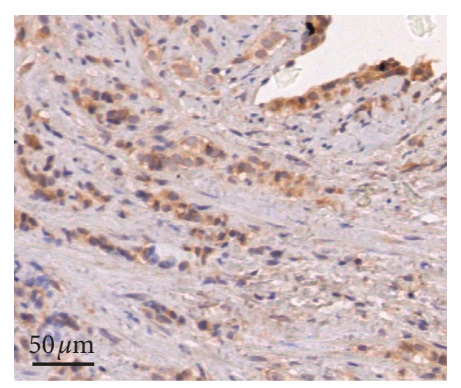

(e)

BM-

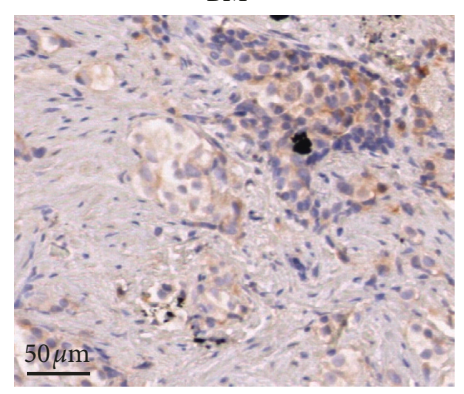

(h)

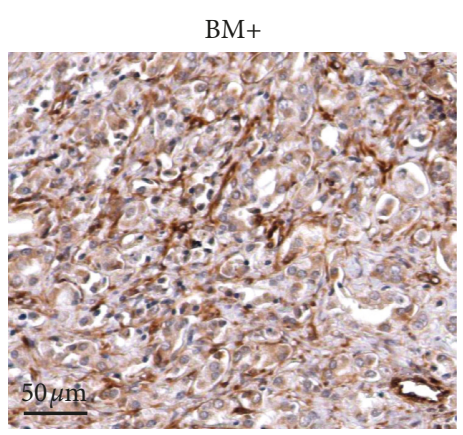

(c)

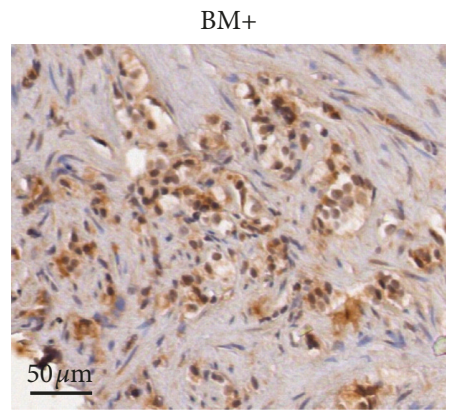

(f)

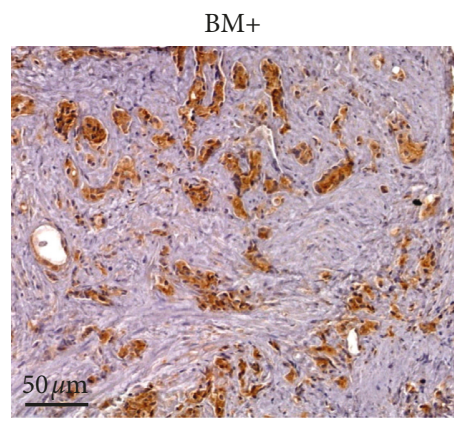

(i)

FIGURE 1: Immunohistochemical analysis of vimentin, BMP-2, and PTX-3. (a) Graph shows the number of vimentin-positive prostate cells in BL, BM+, and BM- lesions. (b) Vimentin-positive prostate cancer cells in BM- lesions (scale bar represents $50 \mu \mathrm{m}$ ). (c) Image shows numerous vimentin-positive prostate cancer cells in BM+ lesions (scale bar represents $50 \mu \mathrm{m}$ ). (d) Graph shows the number of BMP-2positive prostate cells in $\mathrm{BL}, \mathrm{BM}+$, and $\mathrm{BM}$ - lesions. (e) BM+ lesion displaying numerous BMP-2-positive cancer cells (scale bar represents $50 \mu \mathrm{m}$ ). (f) BMP-2-positive prostate cancer cells in BM+ lesions (scale bar represents $50 \mu \mathrm{m}$ ). (g) Graph shows the number of PTX-3-positive prostate cells in $\mathrm{BL}, \mathrm{BM}+$, and BM- lesions. (h) Rare PTX-3-positive cells in BM- lesions (scale bar represents $50 \mu \mathrm{m}$ ). (i) Image shows several PTX-3-positive prostate cancer cells in BM+ (scale bar represents $50 \mu \mathrm{m}$ ).

significantly upregulated in CRPC compared to primary tumours. Interestingly, however, the small set of genes was able to discriminate most of the primary tumours from metastatic CRPCs in unsupervised clustering (Figure 3(b)). Furthermore, analysis of a second dataset with annotated metastatic sites showed that the gene set expression was remarkably higher in tumour specimens taken from bone metastases compared to primary tumours and other metastatic sites (Figure 3(c)).

3.6. Prostate Calcifications. In order to verify if the presence of prostate microcalcifications was linked to the expression of mineralization factors, we subdivided our samples in prostate lesions with (Micro+) or without (Micro-) calcifications, independently from the type of lesion. We observed significantly higher expression of BMP-2 in Micro+ respect to Micro- (Micro+ $431.60 \pm 23.35$ vs Micro- 288.30 $\pm 18.00 ; p=0.0017$ ) (Figure 4(a)). We found an increase of PTX-3-positive prostate cells in Micro+ as compared to Micro- (Micro+ $234.20 \pm 18.40$ vs Micro- $120.7 \pm 25.82$; $p=0.0045$ ) (Figure 4(b)). Conversely, no significant difference was observed for the analysis of RUNX2-positive prostate cells (Micro+ $331.50 \pm 20.52$ vs Micro- $281.50 \pm$ 26.65; $p=0.1980$ ) (Figure 4(c)). Analysis of RANKL showed a significant difference between the presence of RANKLpositive prostate cells between Micro+ and Micro- (Micro+ $283.70 \pm 24.23$ vs Micro- $216.60 \pm 18.07 ; p=0.0252$ ) 


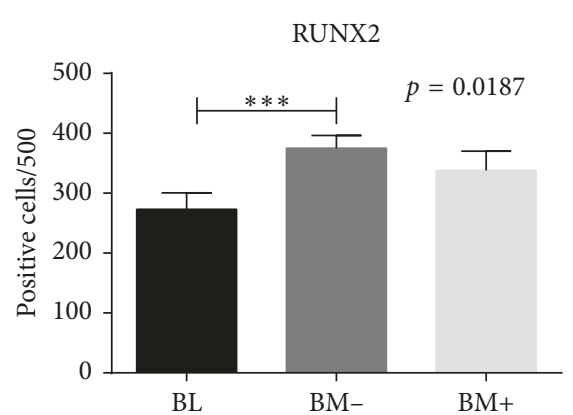

(a)

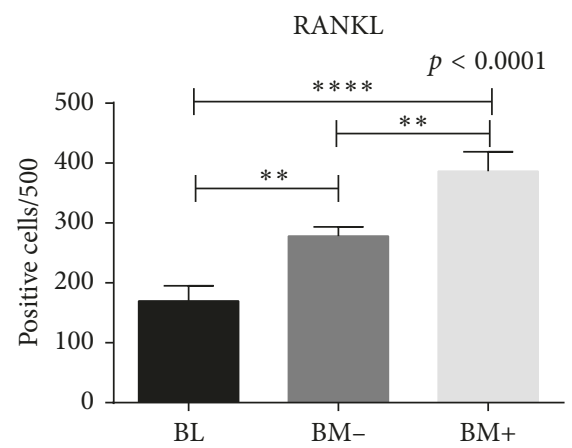

(d)

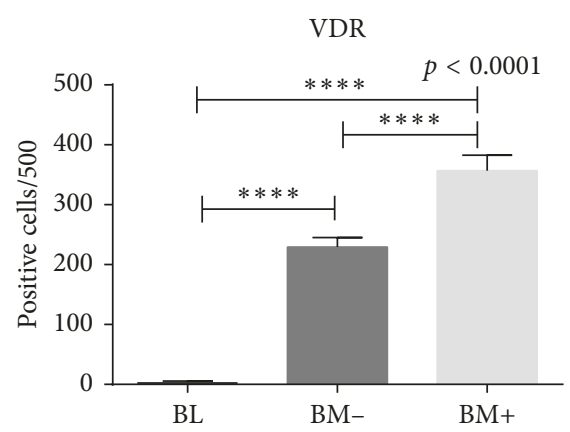

(g)

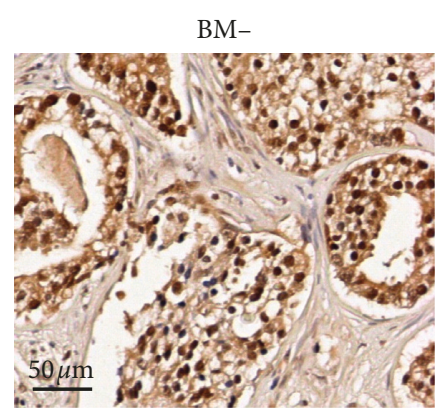

(b)

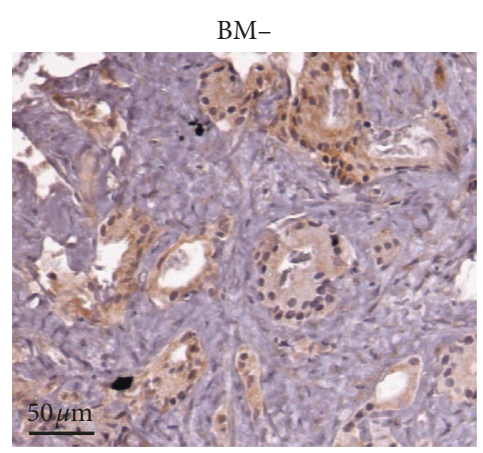

(e)

BM-

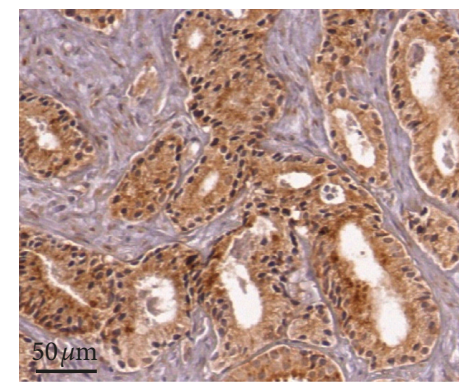

(h)

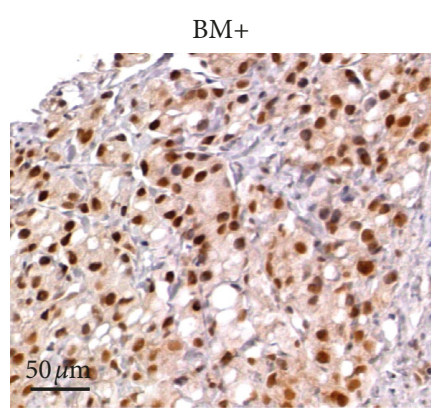

(c)

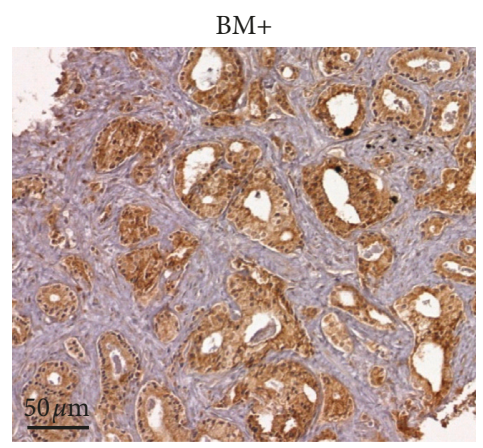

(f)

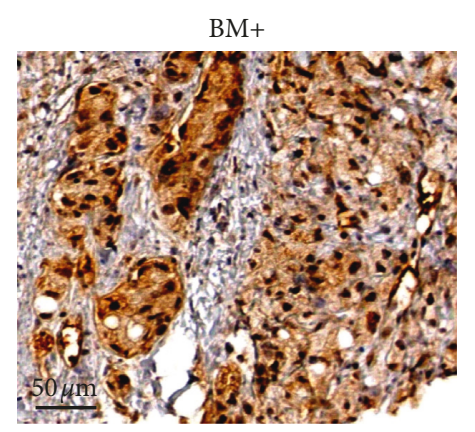

(i)

FIGURE 2: Expression of bone markers in prostate cells. (a) Graph shows the number of RUNX2-positive prostate cells in BL, BM+, and BMlesions. (b) Numerous nuclear RUNX2-positive cancer cells in BM- lesions (scale bar represents $50 \mu \mathrm{m}$ ). (c) Nuclear RUNX" expression in prostate cancer cells of a BM+ patient (scale bar represents $50 \mu \mathrm{m}$ ). (d) Graph displays the number of RANKL-positive prostate cells in BL, $\mathrm{BM}-$, and BM+ lesions. (e) RANKL expression in a case of BM- patient (scale bar represents $50 \mu \mathrm{m}$ ). (f) Numerous prostate cancer cells expressing RANKL in BM+ (scale bar represents $50 \mu \mathrm{m}$ ). (g) Graph shows the number of nuclear VDR-positive prostate cells in BL, BM-, and BM+ lesions. (h) VDR-positive prostate cancer cells in a BM- lesion (scale bar represents $50 \mu \mathrm{m}$ ). (i) Several nuclear VDR-positive prostate cancer cells in a BM+ lesion (scale bar represents $50 \mu \mathrm{m}$ ).

(Figure 4(d)). Finally, significant differences in VDR expression were observed (Micro+ $192.00 \pm 18.02$ vs Micro$109.30 \pm 19.14 ; p=0.001$ ) (Figure $4(\mathrm{e})$ ).

\subsection{Ultrastructural Characterization of Prostate Cancer Cells.} TEM analysis allowed us to characterize ultrastructure of prostate cells in malignant lesions. Specifically, we observed both cuboidal and large spindle-shaped cells with abundant clear cytoplasm in BM+ (Figure 5(a)). Moreover, in these lesions, we identified several calcifications and prostate cancer cells with morphological appearance of osteoblasts containing cytoplasmic electrondense granules made of HA (Figure 5(b)). In addition, EDX microanalysis demonstrated that all calcifications here detected were made of calciumphosphate (hydroxyapatite) (Figure 5(b)).

3.8. 18F-Choline PET/CT Analysis. We collected PET/CT data of 11 patients: $5 \mathrm{BM}+$ and $6 \mathrm{BM}-$ (Figure 5(c)). Despite the low number of patients, we found significant differences between both SUV max and SUV average between BM+ and BM- (Figure 5(d), 5(e)). Noteworthy, the cancer lesions with higher value of SUV max (BM+ patients) (Figure 5(f)) were characterized by the presence of calcium-phosphate calcifications and a higher number $(>300)$ of RUNX2-positive (Figure 5(g)) and RANKL-positive (Figure 5(h)) prostate cancer cells. 

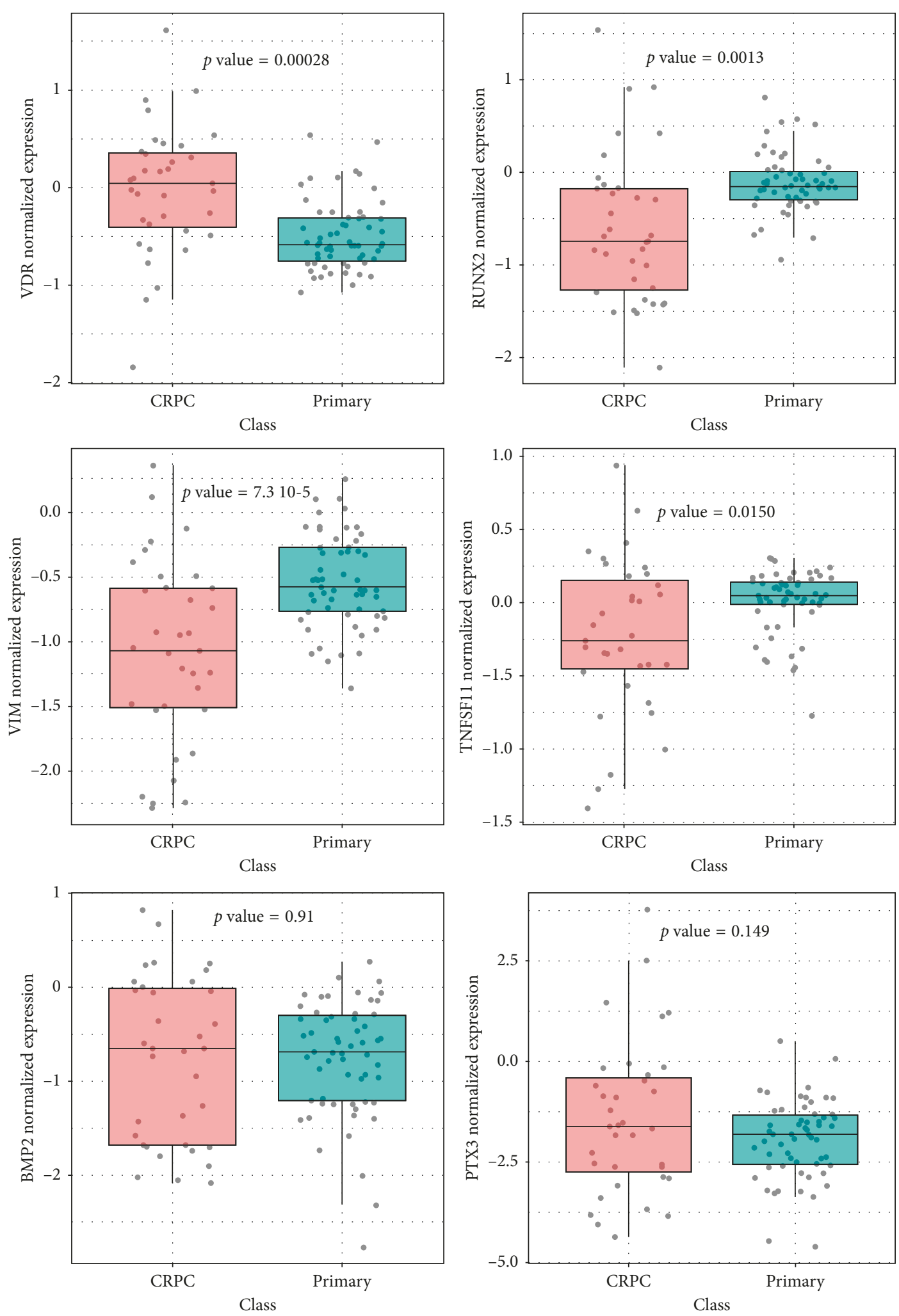

(a)

FIgURE 3: Continued. 


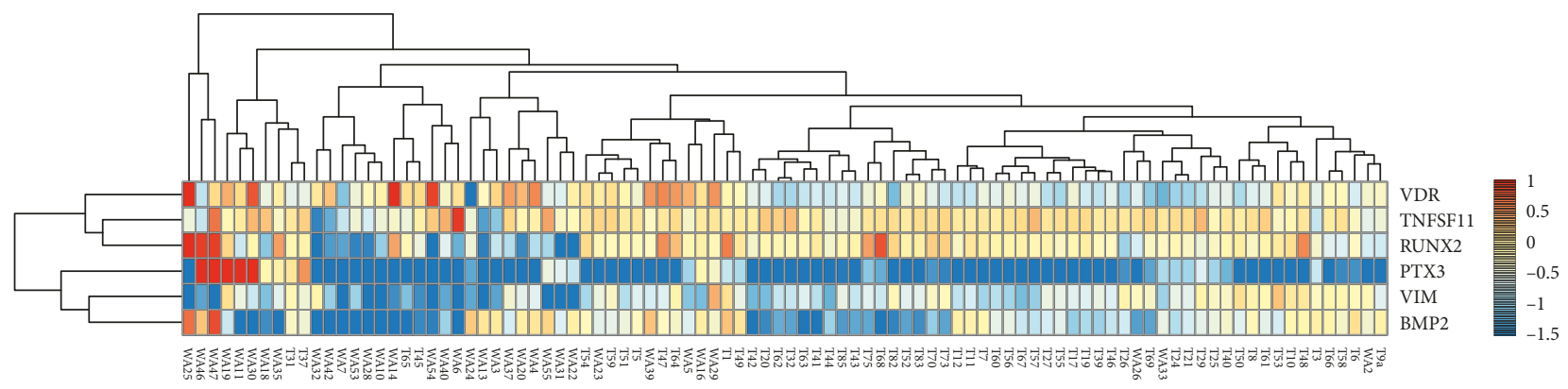

(b)

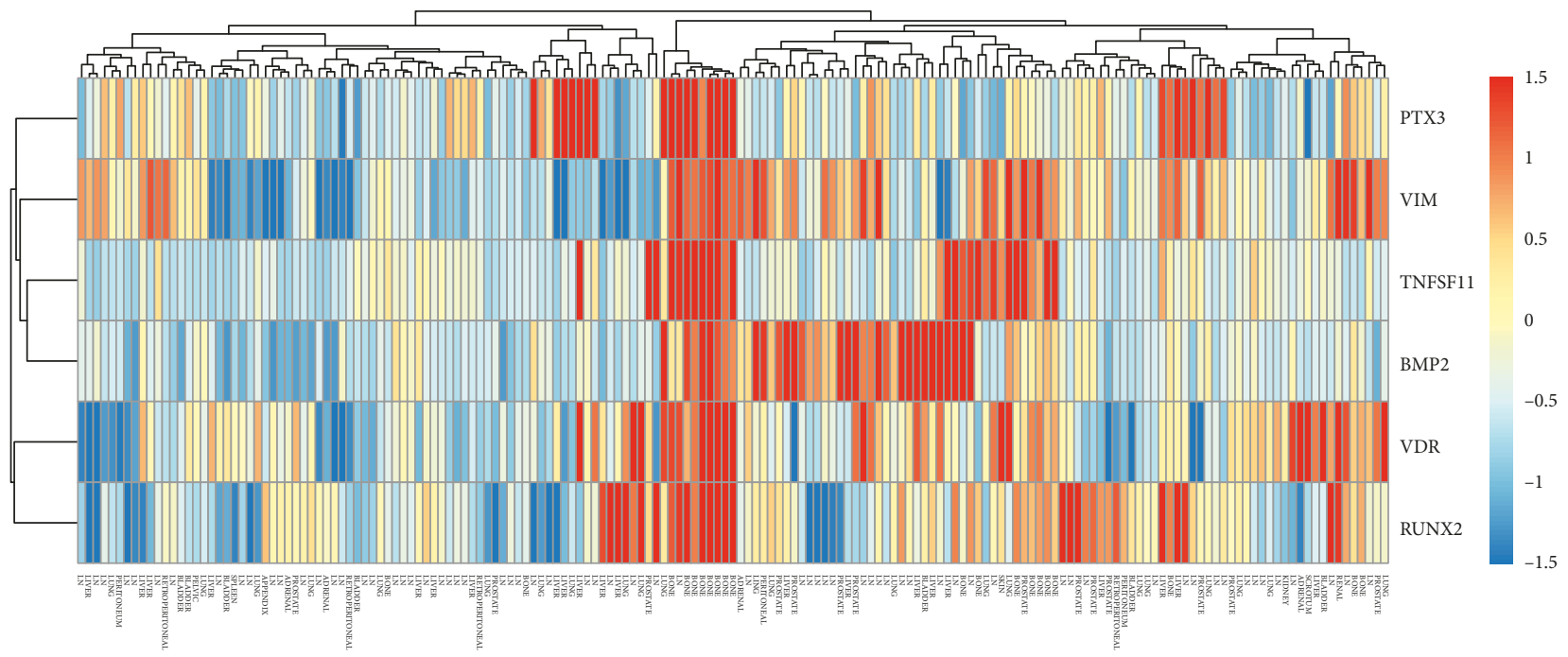

(c)

FIGURE 3: Expression of bone markers in prostate cancer patient datasets. (a) Graphs show the mRNA levels of the genes VDR, RUNX2, vimentin, TNFSF11, BMP-2, and PTX3 in metastatic castration resistant prostate cancer (CRPC) and primary prostate tumours (primary). (b) Unsupervised hierarchical clustering of metastatic (WA) and primary (T) prostate cancers based on expression of the indicated gene set. Metastatic samples are labelled in red; primary samples are labelled in black. (c) Unsupervised hierarchical clustering of primary (prostate) and metastatic prostate cancers at the indicated distinct metastatic sites. Primary/localized samples are indicated in black; distal metastases are indicated in red.

\section{Discussion}

Prostate metastasis to the bone more often results in osteoblastic lesions, though it is known that prostate bone metastases can display both blastic and lytic characteristics during the early phases of their formation [25]. In addition, there is evidence that during the early phases of osteoblastic metastases formation, it is possible to observe osteolytic lesions, suggesting an overall increase of bone remodeling at these sites. The pathophysiology of bone metastases is frequently explained by the theory of the vicious cycle proposed for the first time by Mundy and Guise [26]. According to this theory, cancer cells resident in bone cause bone destruction because they are capable to stimulate osteoclast activity. In return, cancer cells receive positive feedbacks from humoral factors released by the bone microenvironment during bone destruction and remodeling [27]. Indeed, it is widely accepted that the bone microenvironment is crucial to the success of cancer cells in bone.

In a recent study, we described for the first time the characteristics of prostate cells involved in the production of prostate calcifications demonstrating their similarity with osteoblasts [24]. In addition, our research group described the presence of osteoblast-like cells in breast cancer (BOLCs) showing a correlation between the appearance of BOLCs in primary lesions and development of bone metastases. Based on these studies, the main aim of this study was to investigate the possible correlation between the presence of prostate cancer cells showing expression of typical morphological and molecular markers of osteoblasts and the development of bone metastasis in prostate cancer patients within 5 years from diagnosis of primary lesion. To this end, we collected 110 prostate biopsies (44 benign and 66 malignant lesions). Malignant lesions were subdivided in biopsies from patients with clinical evidence of bone metastasis $(\mathrm{BM}+, n=23)$ and those from patients without clinical evidence of bone metastasis (BM-, $n=43$ ).

As already reported by Scimeca et al., we found a significant correlation between vimentin expression, one of the most important markers of mesenchymal cells [14], and the presence of prostate osteoblast-like cells (POLCs). Specifically, our data showed a significant increase of positive cells 


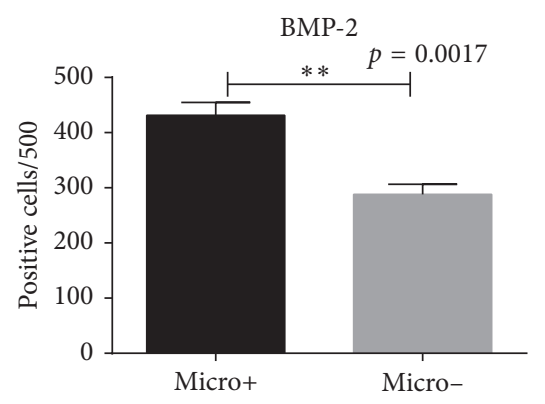

(a)

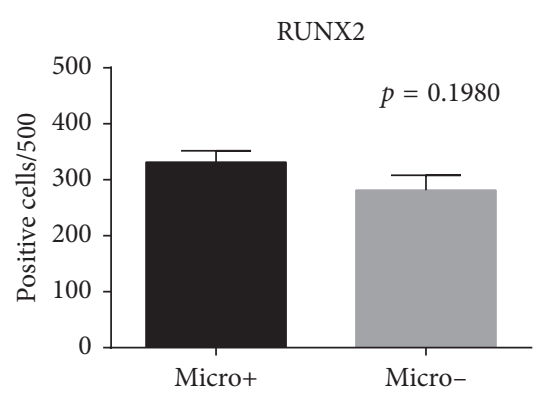

(c)

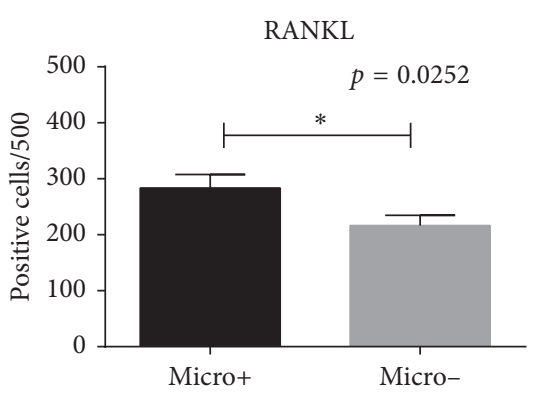

(d)

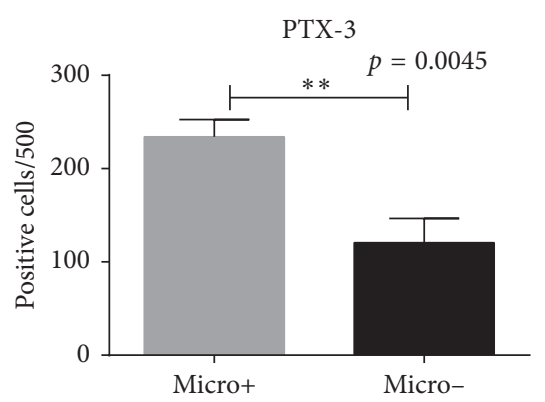

(b)

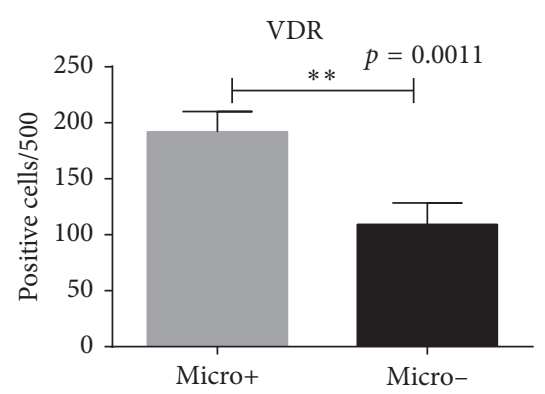

(e)

FIGURE 4: Expression of bone markers in prostate lesions with or without calcification. (a) Graph shows the number of BMP-2-positive prostate cells in Micro+ and Micro- lesions. (b) Graph displays the number of PTX-3-positive prostate cells in Micro- and Micro+ lesions. (c) Graph shows the number of RUNX2-positive prostate cells in Micro+ and Micro-lesions. (d) Graph displays the number of RANKL-positive prostate cells in Micro+ and Micro-lesions. (e) Graph shows the number of VDR-positive prostate cells in Micro+ and Micro- lesions.

in prostate cancer of $\mathrm{BM}+$ group as compared with $\mathrm{BM}-$. In addition, we proved that primary prostate cancer lesions of $\mathrm{BM}+$ patients were characterized by the expression of osteogenic molecules able to induce osteoblast differentiation and to increase osteoblast function such as mineralization. Among them, BMP-2 is a potent inducer of bone formation through the stimulation of osteoblast differentiation. BMP-2 exerts this effect via two types of serine/threonine kinase receptors: BMP-2 binds the type II receptor, which subsequently activates the type I receptor by a direct association [28]. Our results showed an increase of BMP-2 expression in prostate malignant lesions. Conversely, the absence of significant differences of BMP-2 expression between $\mathrm{BM}+$ and $\mathrm{BM}$ - suggests that it could be involved in the early phases of cancer transformation rather than during metastatic process. In support of this, several studies demonstrated the ability of BMP-2 to induce malignant transformation of epithelial tissues [29-31]. However, we also demonstrated the association between BMP-2 expression and the presence of prostate calcifications, regardless of the lesion type. Thus, BMP-2, in association to EMT phenomenon, can participate to induce mesenchymal-like cells to acquire osteoblast phenotype. As concerns PTX-3, PTX-3 is a multifunctional glycoprotein produced by a variety of cells [32, 33]; our results displayed a significant correlation between the presence of PTX-3-positive prostate cells and bone metastasis formation. Also, it is important to emphasize that BMgroup showed the same number of PTX-3-positive cells of $\mathrm{BL}$, suggesting that the presence of PTX-3-positive cells could represent a reliable predictive element for the development of bone metastasis from prostate cancer.
These data are in line with recent studies that demonstrated the involvement of PTX-3 in osteoblast proliferation, differentiation and function [34-36], and bone metastasis from breast cancer formation.

To further characterize the phenotype of POLCs, we investigate the expression of the main markers of osteoblasts, RUNX2, RANKL, and VDR. RUNX2 is the first transcription factor required for the determination of the osteoblast lineage [37]. In particular, RUNX2 is detected first in preosteoblasts and its expression is upregulated during the early phases of osteoblast differentiation. In line with this, our results displayed an increase of RUNX2-positive prostate cells in malignant lesions respect to $\mathrm{BL}$, but no difference was observed between $\mathrm{BM}+$ and $\mathrm{BM}-$. Therefore, the acquisition of RUNX2 expression by prostate cells seems to be linked to cancer transformation rather than to metastatic process.

In agreement with the physiological role of RUNX2 in osteoblast function [38], we did not observe an increase of RUNX2-positive cells in Micro+ with respect to Micro- lesions. Indeed, mature osteoblasts lose the expression of RUNX2 during the mineralization phase of bone formation. Conversely, analysis of RANKL and VDR showed a putative correlation among the presence of RANKL and/or VDR positive prostate cancer cells, bone metastasis formation, and microcalcifications. As regards the formation of bone metastasis, the presence of RANKL-positive prostate cancer cells can trigger osteoclast activity by binding to the osteoclast receptor RANK [39]. Indeed, RANKL is a type II membrane protein expressed by osteoblasts that is able to induce osteoclasts proliferation and function. In addition, at the primary lesion site, RANKL expression can reflect the presence of cells 


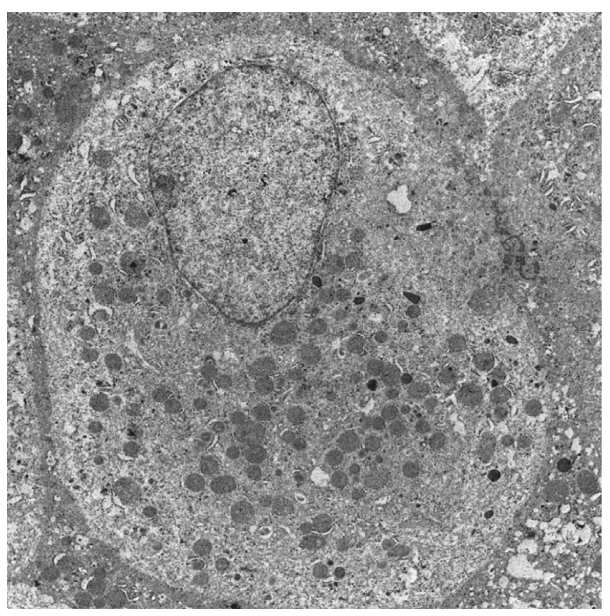

(a)

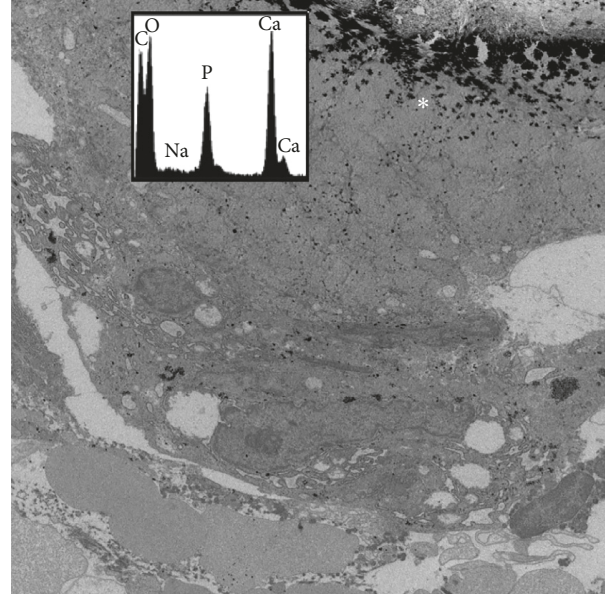

(b)

\begin{tabular}{lccc}
\hline & SUV max & SUV average & $p$ value \\
\hline BM+ & $34.21 \pm 3.3$ & $22.83 \pm 3.35$ & 0.0303 \\
BM- & $13.00 \pm 2.64$ & $9.15 \pm 0.95$ & 0.0442 \\
\hline
\end{tabular}

(c)

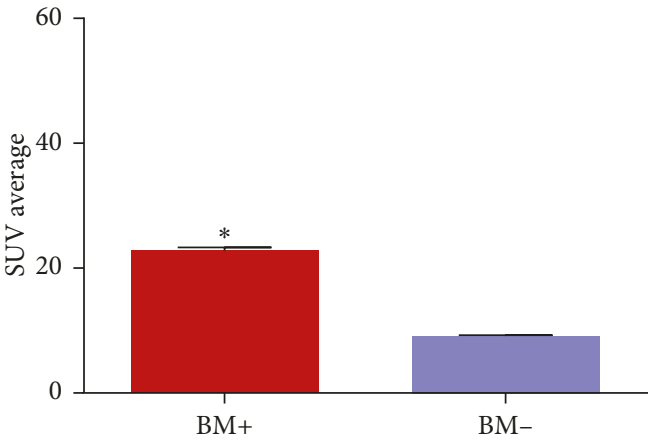

(d)

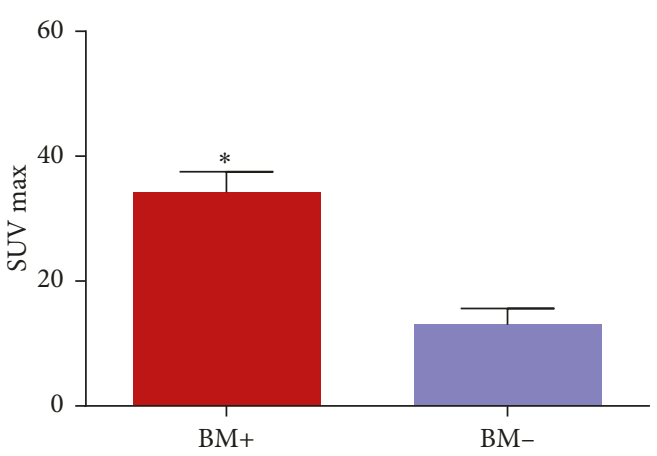

(e)

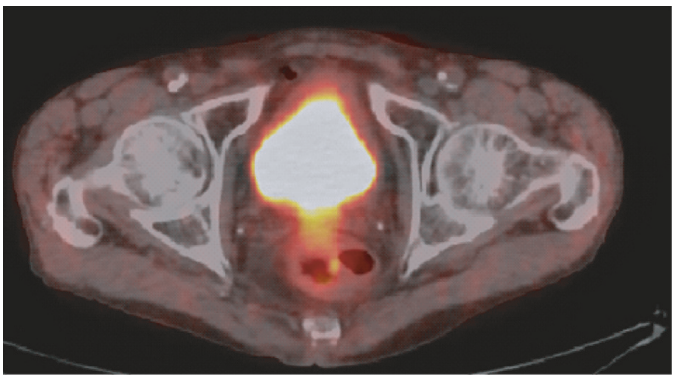

(f)

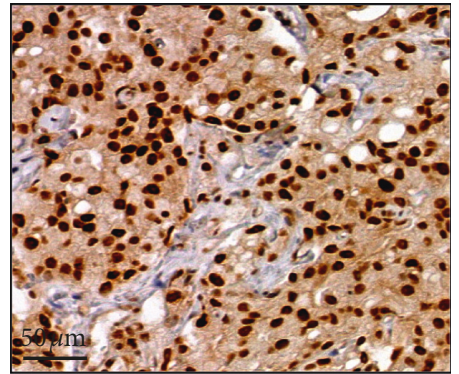

(g)

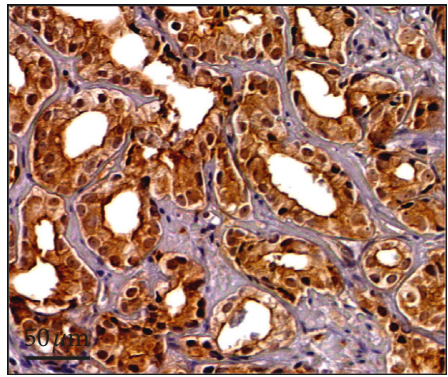

(h)

Figure 5: Ultrastructural and molecular imaging analysis. (a) Electron micrograph shows prostate cancer cells of a BM- biopsy. (b) Prostate cancer cells next to calcium-phosphate calcification in a BM+ lesion. SUV max and SUV average of BM+ and BM- lesions. (c) Graph shows significant difference between the SUV max value of BM+ and BM-patients. (d) Graph shows significant difference between the SUV average value of BM+ and BM- patients. (e) Graph shows significant difference between the SUV max value of BM+ and BM- patients. (f) Dual fusion 18F-choline PET/CT image of BM+ patients. (g) Image displays numerous RUNX2-positive prostate cancer cells in BM+ patient of (e) (scale bar represents $50 \mu \mathrm{m}$ ). (h) Image displays numerous RANKL-positive prostate cancer cells in BM+ patient of (e) (scale bar represents $50 \mu \mathrm{m})$.

responsible for microcalcification production. Similar to what occurs during bone mineralization, our data support the hypothesis that the nuclear translocation of VDR participates in production of microcalcifications in prostate lesions. Thus, nuclear translocation of VDR could be considered a marker of
POLCs since it could be linked to bone metastasis formation. Notably, nuclear VDR is the only protein that we did not find expressed in BL, among all proteins studied here. This evidence candidates nuclear VDR as a reliable prognostic and/or predictive marker of prostate cancer occurrence. Combined 
analysis of this set of genes in patients with primary and metastatic prostate cancer further showed that deregulated expression of these markers of EMT, bone mineralization, and osteoblastic differentiation occurred preferentially in the setting of metastatic disease and particularly at metastases in bone, further supporting their relevance as adverse prognostic markers.

It is important to highlight that in this study, POLCs were also characterized from the ultrastructural point of view. In particular, we observed the presence of cytoplasmic vesicles containing $\mathrm{HA}$ granules in prostate cancer cells showing osteoblast phenotype (POLCs) [24, 40-42].

Of note, although preliminary, our data showed a significant correlation between the uptake of 18F-choline PET/ $\mathrm{CT}$ and the presence of POLCs in prostate cancer tissues. If confirmed in a larger patient cohort, this evidence could provide the scientific rationale for the development of algorithms able to predict the metastatic potential of primary prostate cancer lesions by $18 \mathrm{~F}$-choline PET/CT analysis [43].

This study proposes a new cell type generated by a process of cell transdifferentiation and related to formation of bone metastasis: the POLCs. Although our data require further investigations about the molecular mechanisms of both POLCs generation and metastasization to the bone, this study opens new and interesting prospective for the management of prostate cancer patients. The presence of POLCs could become prognostic markers for occurrence of bone metastatic disease.

\section{Conclusion}

The clinical course of metastatic bone disease in prostate cancers is often long, with patients experiencing sequential skeletal complications over a period of several years. These include bone pain, fractures, hypercalcemia, and spinal cord compression, all of which may profoundly impair patient's quality of life. In addition, once prostate tumour cells are engrafted in the skeleton, curative therapy is no longer possible and palliative treatment becomes the only option. Thus, the identification of early markers of bone metastasis and especially the characterization of the cells involved in the metastatic process can lay the foundation for the identification of new tools for monitoring, prevention, or cure of bone metastatic diseases and providing support to the physicians in the management of prostate patients. In this context, positron emission tomography (PET)/computed tomography (CT) has emerged as a significant and promising staging modality for primary, recurrent, and metastatic prostate cancer. Much more important, the identification of highly sensitive and specific radiotracers can implement the therapeutic/diagnostic perspectives for prostate cancer patients "opening the way" for the development of new theranostic approaches. PSMA PET/ $\mathrm{CT}$ ligands labelled with ${ }^{18} \mathrm{~F}$ and ${ }^{68} \mathrm{Ga}$ have certainly revolutionized the management of metastatic prostate cancer selecting patients who may benefit from targeted systemic radionuclide therapy. In a nuclear oncology theranostic design, ${ }^{68} \mathrm{Ga}$-PSMA already constitutes the diagnostic positronemitting of beta ${ }^{-}$emitter Lutetium-177 PSMA ( ${ }^{177}$ Lu-PSMA) [44] and alpha-emitter Actinium-225 PSMA ( ${ }^{225}$ Ac-PSMA)
[45]. Finally, the results reported here about the phenotypic characterization of POLCs could provide a scientific rationale for the development of theranostic anti-POLC radiomolecules for the cure and prevention of prostate cancer bone metastasis.

\section{Data Availability}

The data used to support the findings of this study are included within the article. Gene expression data from two studies in prostate cancer patients (reference [21] and [22]) were retrieved from the cBioPortal platform. Expression of the selected genes was compared between primary tumours and metastatic CRPC and, for the second dataset, among primary and different metastatic sites.

\section{Conflicts of Interest}

The authors declare that there are no potential conflicts of interest relating to the manuscript.

\section{Acknowledgments}

The authors wish to thank Dr. Sara Fazi, Dr. Alessandro Polidori, and Dr. Serena Chiantini for technical support.

\section{References}

[1] C. L. Eaton and R. E. Coleman, "Pathophysiology of bone metastases from prostate cancer and the role of bisphosphonates in treatment," Cancer Treatment Reviews, vol. 29, no. 3 , pp. 189-98, 2003.

[2] S. Sethi, J. Macoska, W. Chen, and F. H. Sarkar, "Molecular signature of epithelial-mesenchymal transition (EMT) in human prostate cancer bone metastasis," American Journal of Translational Research, vol. 3, no. 1, pp. 90-9, 2010.

[3] A. M. Demarzo, W. G. Nelson, W. B. Isaacs, and J. I. Epstein, "Pathological and molecular aspects of prostate cancer," The Lancet, vol. 361, no. 9361, pp. 955-964, 2003.

[4] S. De, J. Chen, N. V. Narizhneva et al., "Molecular pathway for cancer metastasis to bone," Journal of Biological Chemistry, vol. 278, no. 40, pp. 39044-39050, 2003.

[5] J. J. Yin, C. B. Pollock, and K. Kelly, "Mechanisms of cancer metastasis to the bone," Cell Research, vol. 15, no. 1, pp. 57-62, 2005.

[6] T. Martin, L. Ye, A. J. Sanders, J. Lane, and W. G. Jiang, Cancer Invasion and Metastasis: Molecular and Cellular Perspective, Landes Bioscience, Austin, TX, USA, 2000-2013.

[7] I. J. Fidler and M. L. Kripke, "Metastasis results from preexisting variant cells within a malignant tumor," Science, vol. 197, no. 4306, pp. 893-5, 1977.

[8] G. Poste and I. J. Fidler, "The pathogenesis of cancer metastasis," Nature, vol. 283, no. 5743, pp. 139-46, 1980.

[9] Y. Wu, M. Sarkissyan, and J. V. Vadgama, "Epithelialmesenchymal transition and breast cancer," Journal of Clinical Medicine, vol. 5, no. 2, 2016.

[10] M. Scimeca, E. Giannini, C. Antonacci et al., "Microcalcifications in breast cancer: an active phenomenon mediated by epithelial cells with mesenchymal characteristics," BMC Cancer, vol. 14, no. 1, p. 286, 2014.

[11] M. Scimeca, C. Antonacci, and E. Bonanno, "Breast microcalcifications: a focus," Journal of Cell Science and Therapy, vol. S8, p. e101, 2015. 
[12] M. Scimeca, C. Antonacci, N. Toschi et al., "Breast osteoblastlike cells: a reliable early marker for bone metastases from breast cancer," Clinical Breast Cancer, vol. 18, no. 4, pp. e659-e669, 2018.

[13] M. Scimeca, R. Bonfiglio, M. Montanaro, and E. Bonanno, "Osteoblast-like cells in human cancers: new cell type and reliable markers for bone metastasis," Future Oncology, vol. 14, no. 1, pp. 9-11, 2018.

[14] M. Scimeca, C. Antonacci, D. Colombo, R. Bonfiglio, O. C. Buonomo, and E. Bonanno, "Emerging prognostic markers related to mesenchymal characteristics of poorly differentiated breast cancers," Tumor Biology, vol. 37, no. 4, pp. 5427-35, 2016.

[15] R. Bonfiglio, M. Scimeca, N. Toschi et al., "Radiological, histological and chemical analysis of breast microcalcifications: diagnostic value and biological significance," Journal of Mammary Gland Biology and Neoplasia, vol. 23, no. 1-2, pp. 89-99, 2018.

[16] M. Scimeca, S. Bischetti, H. K. Lamsira, R. Bonfiglio, and E. Bonanno, "Energy Dispersive X-ray (EDX) microanalysis: a powerful tool in biomedical research and diagnosis," European Journal of Histochemistry, vol. 62, no. 1, p. 2841, 2018.

[17] M. Scimeca, A. Pietroiusti, F. Milano et al., "Elemental analysis of histological specimens: a method to unmask nano asbestos fibers," European Journal of Histochemistry, vol. 60, no. 1, p. 2573, 2016.

[18] M. Scimeca, A. Orlandi, I. Terrenato, S. Bischetti, and E. Bonanno, "Assessment of metal contaminants in non-small cell lung cancer by EDX microanalysis," European Journal of Histochemistry, vol. 58, no. 3, p. 2403, 2014.

[19] F. Calabria, A. Chiaravalloti, C. Cicciò et al., "PET/CT with 18F-choline: physiological whole bio-distribution in male and female subjects and diagnostic pitfalls on 1000 prostate cancer patients: 18F-choline PET/CT bio-distribution and pitfalls. A southern Italian experience," Nuclear Medicine and Biology, vol. 51, pp. 40-54, 2017.

[20] F. Calabria, D. Rubello, and O. Schillaci, "The optimal timing to perform $18 \mathrm{~F} / 11 \mathrm{C}$-choline $\mathrm{PET} / \mathrm{CT}$ in patients with suspicion of relapse of prostate cancer: trigger PSA versus PSA velocity and PSA doubling time," International Journal of Biological Markers, vol. 29, no. 4, pp. e423-30, 2014.

[21] C. S. Grasso, Y. M. Wu, D. R. Robinson et al., "The mutational landscape of lethal castration-resistant prostate cancer," Nature, vol. 487, no. 7406, pp. 239-43, 2012.

[22] A. Kumar, I. Coleman, C. Morrissey et al., "Substantial interindividual and limited intraindividual genomic diversity among tumors from men with metastatic prostate cancer," Nature Medicine, vol. 22, no. 4, pp. 369-78, 2016.

[23] N. Mottet, J. Bellmunt, M. Bolla et al., "EAU-ESTRO-SIOG Guidelines on prostate cancer. Part 1: screening, diagnosis, and local treatment with curative intent," European Urology, vol. 71, no. 4, pp. 618-629, 2017.

[24] M. Scimeca, R. Bonfiglio, F. Varone, S. Ciuffa, A. Mauriello, and E. Bonanno, "Calcifications in prostate cancer: an active phenomenon mediated by epithelial cells with osteoblastphenotype," Microscopy Research and Technique, vol. 81, no. 7, pp. 745-748, 2018.

[25] S. Ziaee, G. C. Chu, J. M. Huang, S. Sieh, and L. W. Chung, "Prostate cancer metastasis: roles of recruitment and reprogramming, cell signal network and three-dimensional growth characteristics," Translational Andrology and Urology, vol. 4, no. 4, pp. 438-54, 2015.

[26] T. A. Guise and G. R. Mundy, "Cancer and bone," Endocrine Reviews, vol. 19, no. 1, pp. 18-54, 1998.
[27] S. Casimiro, A. R. Ferreira, A. Mansinho, I. Alho, and L. Costa, "Molecular mechanisms of bone metastasis: which targets came from the bench to the bedside?," International Journal of Molecular Sciences, vol. 17, no. 9, 2016.

[28] T. Ogasawara, H. Kawaguchi, S. Jinno et al., "Bone morphogenetic protein 2-induced osteoblast differentiation requires Smad-mediated down-regulation of Cdk6," Molecular and Cellular Biology, vol. 24, no. 15, pp. 6560-8, 2004.

[29] P. Huang, A. Chen, W. He et al., "BMP-2 induces EMT and breast cancer stemness through $\mathrm{Rb}$ and CD44," Cell Death Discovery, vol. 3, p. 17039, 2017.

[30] J. Spanjol, G. Djordjević, D. Markić, M. Klarić, D. Fuckar, and D. Bobinac, "Role of bone morphogenetic proteins in human prostate cancer pathogenesis and development of bone metastases: immunohistochemical study," Collegium Antropologicum, vol. 34, no. 2, pp. 119-25, 2010.

[31] B. Herrera, S. Dooley, and K. Breitkopf-Heinlein, "Potential roles of bone morphogenetic protein (BMP)-9 in human liver diseases," International Journal of Molecular Sciences, vol. 15, no. 4, pp. 5199-220, 2014.

[32] F. Moalli, S. Jaillon, A. Inforzato et al., "Pathogen recognition by the long pentraxin PTX3," Journal of Biomedicine and Biotechnology, vol. 2011, Article ID 830421, 15 pages, 2011.

[33] C. Cappuzzello, A. Doni, E. Dander et al., "Mesenchymal stromal cell-derived PTX3 promotes wound healing via fibrin remodeling," Journal of Investigative Dermatology, vol. 136, no. 1, pp. 293-300, 2016.

[34] M. Scimeca, A. Salustri, E. Bonanno et al., "Impairment of PTX3 expression in osteoblasts: a key element for osteoporosis," Cell Death and Disease, vol. 8, no. 10, p. e3125, 2017.

[35] U. Tarantino, M. Feola, M. Celi, and M. Scimeca, "PTX3: a new mediator of bone metabolism and osteoporosis," Muscle, Ligaments and Tendons Journal, vol. 7, no. 1, pp. 200-201, 2017.

[36] D. Grčević, M. Sironi, S. Valentino et al., "The long pentraxin 3 plays a role in bone turnover and repair," Frontiers in Immunology, vol. 9, p. 417, 2018.

[37] T. Komori, "Regulation of osteoblast differentiation by Runx2," Advances in Experimental Medicine and Biology, vol. 658, pp. 43-9, 2010.

[38] T. Komori, "Roles of Runx2 in skeletal development," Advances in Experimental Medicine and Biology, vol. 962, pp. 83-93, 2017.

[39] J. H. Kim and N. Kim, "Signaling pathways in osteoclast differentiation," Chonnam Medical Journal, vol. 52, no. 1, pp. 12-7, 2016.

[40] B. Ecarot-Charrier, N. Shepard, G. Charette, M. Grynpas, and F. H. Glorieux, "Mineralization in osteoblast cultures: a light and electron microscopic study," Bone, vol. 9, no. 3, pp. 147-154, 1988.

[41] C. R. Howlett, "The fine structure of the proximal growth plate of the avian tibia," Journal of Anatomy, vol. 128, pp. 377-399, 1979.

[42] M. Rohde and H. Mayer, "Exocytotic process as a novel model for mineralization by osteoblasts in vitro and in vivo determined by electron microscopic analysis," Calcified Tissue International, vol. 80, no. 5, pp. 323-336, 2007.

[43] M. Scimeca, N. Urbano, R. Bonfiglio, O. Schillaci, and E. Bonanno, "Management of oncological patients in the digital era: anatomic pathology and nuclear medicine teamwork," Future Oncology, vol. 14, no. 11, pp. 1013-1015, 2018.

[44] J. Ferdinandus, J. Violet, S. Sandhu, and M. S. Hofman, "Prostate-specific membrane antigen theranostics: therapy 
with lutetium-177," Current Opinion in Urology, vol. 28, no. 2, pp. 197-204, 2018.

[45] S. Kojima, J. M. Cuttler, N. Shimura, H. Koga, A. Murata, and A. Kawashima, "Present and future prospects of radiation therapy using a-emitting nuclides," Dose Response, vol. 16, no. 1, article 1559325817747387, 2018. 


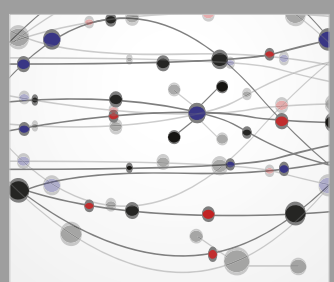

The Scientific World Journal
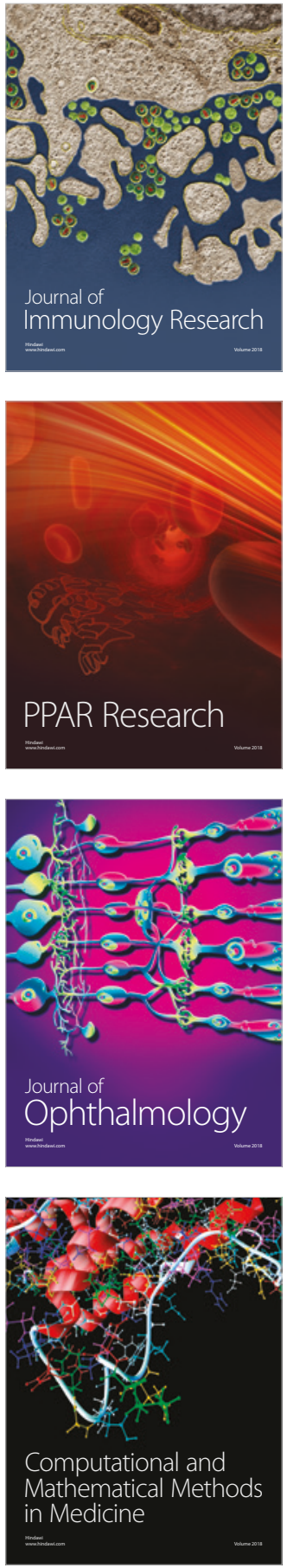

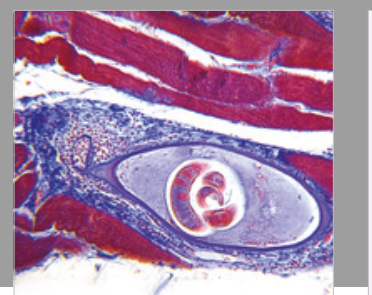

Gastroenterology Research and Practice

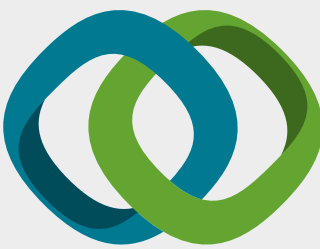

\section{Hindawi}

Submit your manuscripts at

www.hindawi.com
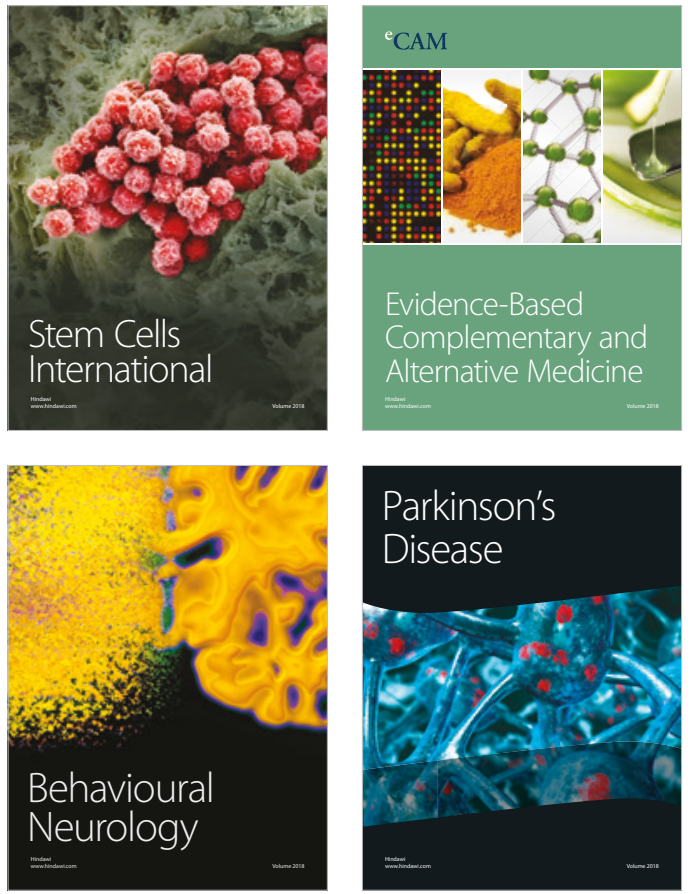

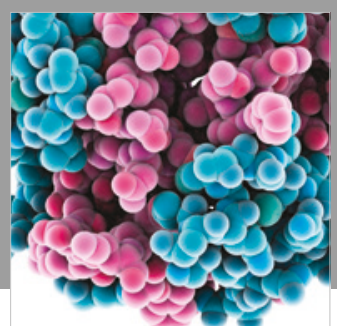

ournal of

Diabetes Research

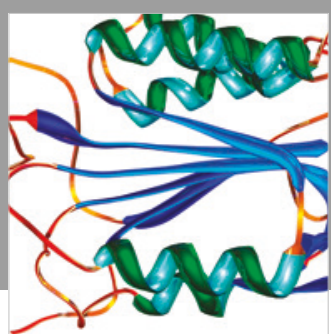

Disease Markers
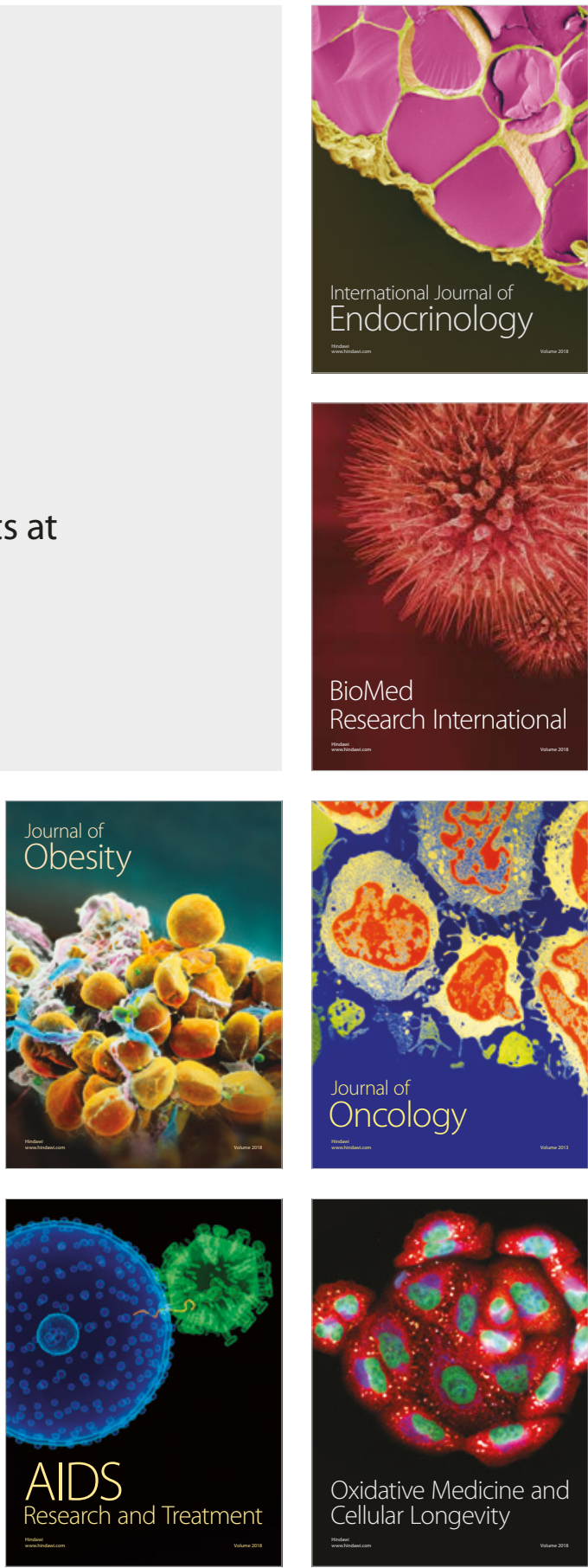